\title{
EDITORIAL
}

\section{Excess mortality in patients with asthma on long-acting $\beta_{2}$-agonists}

\author{
J. Hasford* and J.C. Virchow ${ }^{\#}$
}

$\mathbf{A}$ sthma ranks among the most common of chronic diseases in the Western world. Although modern therapy has improved patient care, asthma still affects $5-10 \%$ of the population and, according to the World Health Organization, claims the lives of some 12,000 patients per year in Western Europe [1]. $\beta_{2}$-Agonists are potent bronchodilators and are the most widely prescribed drugs for asthma worldwide. National and international guidelines recommend shortacting $\beta_{2}$-agonists as as-needed therapy for symptomatic relief in all stages of asthma. Long-acting $\beta_{2}$-agonists (LABAs) have been recommended as controller medication for moderate and severe asthma. Nevertheless, the safety profile of short-acting $\beta_{2}$-agonists has been questioned due to possible detrimental effects on asthma control [2-5]. Recent evidence and metaanalysis suggest an increased risk for cardiovascular complications in patients using $\beta_{2}$-agonists [6-8] and, in addition, there is evidence suggesting that the frequent use of these drugs might increase the risk of premature death [9-11]. The hypothesis that $\beta_{2}$-agonists can have fatal adverse effects was first demonstrated in the late 1960s [12]. INMAN and ADELSTEIN [12] reported a 30-700\% increase, depending on age, in asthma death in patients using pressurised aerosols containing, most often, isoprenaline. The excess mortality in patients with asthma at that time was attributed to overdosing (metereddose aerosols were not widely available at the time). $\beta_{2}$-Agonists came into focus again in the 1980s, when more selective $\beta_{2}$-agonists were introduced in metered-dose inhalers. However, in the 1990s, case-control studies showed that the use of these drugs was associated with an increased risk of fatal asthma, even when inhaled from a metered-dose inhaler. A dose-dependent risk of death from asthma was reported, which increased up to 20 -fold with the use of $\beta_{2}$-agonists [11]. LABAs were developed and first marketed in the early 1990s, and it was suggested that these drugs could be free of the fatal adverse drug reactions associated with their short-acting counterparts. Since then, two large randomised, double-blind trials have been performed to test the hypothesis that the use of LABAs in asthmatic patients is associated with an increased risk of death. The second trial, the Salmeterol Multicenter Asthma

\footnotetext{
*Dept of Medical Informatics, Biometry and Epidemiology, Ludwig-Maximilians-Universität, Munich, " Faculty of Medicine, Rostock University Medical Clinic, Dept of Pneumology, Rostock, Germany.
}

CORRESPONDENCE: J. Hasford, IBE - Dept of Medical Informatics, Biometry and Epidemiology, Ludwig-Maximilians-Universität, Marchioninistr. 15, D-81377 München, Germany. Fax: 49 8970957482. E-mail: has@ibe.med.uni-muenchen.de
Research Trial (SMART), has recently been published [13]. The present editorial reviews the evidence provided by these trials, which belongs to evidence class $\mathrm{Ib}$.

The Serevent Nationwide Surveillance (SNS) Study [14] was a 16-week, double-blind, randomised trial to investigate the safety of the LABA salmeterol in comparison with the shortacting $\beta_{2}$-agonist salbutamol. A total of 25,180 patients with asthma were recruited in the UK. Exclusion criteria were as follows: 1) treatment with $\beta$-blockers; 2) severe uncontrolled pulmonary or systemic disease; 3) pregnancy; and 4) $<12$ yrs of age. The SNS study treatment consisted of $50 \mu \mathrm{g}$ salmeterol b.i.d. or $200 \mu \mathrm{g}$ salbutamol q.i.d. plus the required placebo inhalations. Follow-up examinations were performed after 4, 8 and 16 weeks. As the main target parameter was safety, all serious adverse events and drop-outs were recorded. Of the patients, $\sim 50 \%$ were male, $45 \%$ were $>50$ yrs of age, $\sim 17.5 \%$ had severe asthma, and $69 \%$ took inhaled and $4.8 \%$ oral steroids. Ninety-one per cent had used inhaled $\beta_{2}$-agonists in the past. Death due to any cause occurred in $0.32 \%$ of the patients in the salmeterol group (54 out of 16,787 ) and in $0.24 \%$ (20 out of 8,393 ) of the patients in the salbutamol group (relative risk (RR) 1.35, Chi-squared $=1.3, p=0.250$ ). The corresponding figures for death due to asthma or airway disease were $0.07 \%$ (12 out of 16,787 ) and $0.02 \%$ (two out of 8,393 ) (RR 3, p=0.105). The number needed to harm (NNH) for death of any cause was 1,250 in patients treated with salmeterol for 16 weeks. By the standards at the time, the SNS study was well designed and reported. However, the intention-to-treat analysis of the primary safety end-points using the Chi-squared test was inappropriate. Instead, KaplanMeier curves and the log rank test would have been correct, as the event rates should have been calculated only for those patients who were actually at risk. Thus, the true adverse event incidences were underestimated in the SNS study because the denominator included all randomised patients and was not adjusted, e.g. for those 3,256 patients who dropped out prematurely (and thus decreased their risk of reaching $\beta_{2^{-}}$ agonist-associated end-points). The salmeterol dose corresponded to the daily dosage of $100 \mu \mathrm{g}$ recommended in most countries (this dosage is equivalent to the recommended dosage in the USA of $42 \mu \mathrm{g}$ b.i.d.); the $800 \mu \mathrm{g}$ daily dose of salbutamol was at the upper end of the recommended range

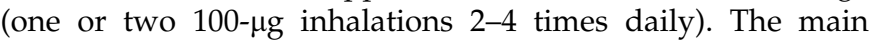
conclusion of this first large-scale, randomised, double-blind trial was that more deaths occurred with salmeterol but, compared with salbutamol, this difference was not statistically significant. Still, the results warrant special attention as this 
trial explicitly investigated whether salmeterol is associated with higher mortality.

Due to the accumulating concerns regarding the safety of LABAs, the Food and Drug Administration (FDA) asked the manufacturer of salmeterol to initiate a large safety trial when it was approved in the USA in 1994. The primary end-point of this randomised, double-blind, placebo-controlled trial, named SMART [13], was to determine either the number of combined respiratory-related deaths or respiratory-related life-threatening experiences (i.e. intubation and mechanical ventilation). Secondary end-points were as follows: 1) deaths due to asthma; 2) combined deaths due to asthma or life-threatening experiences; and 3) deaths of any cause. The observation period was 28 weeks. The SMART study included asthma patients aged $>12$ yrs receiving appropriate treatment. Exclusion criteria were as follows: 1) treatment with $\beta$ blockers; 2) a history of adverse reactions to $\beta$-agonists; and 3) earlier or ongoing treatment with LABAs. The SMART study treatment consisted of $2 \times 42 \mu \mathrm{g}$ of inhaled salmeterol or two inhalations of placebo daily. After a baseline medical examination, patients received the complete medication for the whole treatment period. Follow-up was carried out by telephone after $4,8,12,16,20,24$ and 28 weeks.

An interim analysis was to be performed after $\sim 30,000$ patients were recruited. The protocol called for SMART to be terminated if evidence was found that salmeterol led to an increase in either the primary end-point or asthma-related death. The termination criterion was defined as an RR of 1.4 for the primary end-point and an RR of 3.0 for asthma-related deaths. This interim analysis was performed in 2002 after 25,858 patients had been recruited. SMART was prematurely terminated on January 23, 2003, and 26,355 patients were included in the final analysis (salmeterol $n=13,176$, placebo $n=13,179)$. The following end-points occurred statistically significantly $(\mathrm{p}<0.05)$ more frequently in the salmeterol group: 1) respiratory-related deaths (24 out of 13,176 versus 11 out of 13,179 cases, RR 2.2); 2) combined asthma-related death or lifethreatening experiences (37 versus 22 cases, RR 1.71); and 3) asthma-related deaths (13 versus three cases, RR 4.4). The NNH for death of any cause was 1,316 for patients treated for 28 weeks with salmeterol. In an attempt to explain the results of SMART, the authors, two of them employees of the sponsor, argued that the imbalance of the end-points occurred largely in the African-American subpopulation $(18 \%)$ and in patients who did not use inhaled corticosteroids concurrently. In addition, the majority of asthma-related deaths (13 out of 16) occurred when patients were recruited by advertising, and only three cases were seen (but all three in the salmeterol group) when patients were recruited directly by investigators. Some of these observations may be interesting. However, they all are results of post hoc (i.e. not specified in the trial protocol) subgroup analyses. As the authors state, the results of post hoc analyses are not adequate to draw conclusions [13]. Since patients with systemic diseases that might place a subject at risk, as well as patients with a history of adverse reactions to any sympathomimetic amine drug were excluded, the risks of using LABA under real-life conditions are likely to be even higher than those reported in SMART. SMART confirmed the $a$ priori hypothesis that inhaled salmeterol leads to excess mortality in asthma patients; the SMART study had explicitly addressed this question and it was terminated prematurely. The protocol-defined termination criteria, a RR of 1.4 for the main target parameter and 3.0 for asthma-related deaths in the life-table analysis, had been reached (RR 1.40 and 4.4, respectively). In view of previous findings [14], it would have been unconscientious and unethical to recruit additional patients simply to confirm the excess salmeterol-associated mortality for the primary end-point with statistical significance. Given these results, the FDA ordered a black box warning for all salmeterol-containing products in 2003, stating that a large placebo-controlled trial had shown a small but significant increase in asthma-related deaths in patients receiving salmeterol. To date, no such warning has been issued by the European Agency for the Evaluation of Medicinal Products.

Studies similar in size to SMART are not available for formoterol, which has been approved in the USA in a 12-mg formulation based on two 12-week studies [15, 16] and a 1-yr study in children aged 5-12 yrs [4]. A formoterol $2 \times 12-\mu \mathrm{g}$ puff was compared with a $2 \times 24-\mu \mathrm{g}$ puff per day or placebo with on-demand treatment with short-acting $\beta_{2}$-agonists. In all three studies, the highest number of severe asthma exacerbations, defined as either life threatening, requiring hospital admission, or causing disability or death due to status asthmaticus, occurred in the groups that received the highest dose of formoterol which, in addition, was not clinically superior to the $2 \times 12 \mathrm{mg}$ dose. A phase IV post-marketing study over 16 weeks with 2,085 patients with mild-to-moderate persistent asthma led to five ( $0.9 \%$ of all patients) severe asthma-related complications in the group receiving $24 \mu \mathrm{g}$ formoterol $(n=527)$, but to only two $(0.4 \%)$ in the $12-\mu \mathrm{g}$ group $(n=527)$ and only one $(0.2 \%)$ in the placebo group $(n=514)$ [17]. Therefore, the FDA issued the same warning for formoterol, assuming a class effect.

\section{CONCLUSIONS}

Two large-scale, double-blinded, randomised studies with a total of $>50,000$ patients assessing the safety profile of salmeterol are now available. Both studies explicitly addressed the question as to whether mortality is increased with salmeterol. Both studies clearly showed an excess mortality in the salmeterol group, which in one study (SMART) was statistically significant for several cause-of-death categories. All-cause mortality was almost identical in both trials. The $\mathrm{NNH}$ for all-cause mortality was also highly comparable $(1: 1,250$ versus $1: 1,316)$ and indicates that the risks are of publichealth relevance. In contrast to efficacy studies, according to ethical and legal issues, waiting until predefined significance limits (in SMART $p \leqslant 0.01$ ) are reached in safety studies is not allowed. The imperative of patient protection holds true. In view of the results of the two studies, both of the highest evidence class $(\mathrm{Ib})$, the existence of salmeterol-related excess mortality has to be assumed with near certainty. As the results from a number of smaller studies with formoterol point in the same direction, the effects observed in these safety studies for salmeterol are likely to be identical for formoterol until proven otherwise.

\section{CLINICAL CONSIDERATIONS}

At present, there is little evidence that the addition of inhaled corticosteroids to long-acting $\beta_{2}$-agonists will abolish the risk 
associated with these drugs. Clearly, additional and correctly designed studies with sufficiently large numbers of patients are needed to address these issues. At present, patients with asthma should be initiated and maintained on sufficiently high doses of inhaled corticosteroids and only patients whose asthma cannot be controlled should receive additional longacting $\beta_{2}$-agonists on a regular basis. Whether the addition of a leukotriene-receptor antagonist to moderate-to-high-dose inhaled corticosteroids could be a safe and equally effective alternative will require further long-term studies investigating this option. Until then, the use of these medications should be limited to patients with asthma that cannot be controlled otherwise. In addition, long-acting $\beta_{2}$-agonists should be withdrawn from patients who do not profit from their use. Finally, newly developed ultra-long-acting $\beta_{2}$-agonists, such as indacaterol and others, need to be assessed for this risk prior to approval for widespread use in patients with asthma.

\section{REFERENCES}

1 World Health Organization. The World Health Report 2003. Geneva, WHO, 2004.

2 Sears MR, Taylor DR, Print CG, et al. Regular inhaled betaagonist treatment in bronchial asthma. Lancet 1990; 336: 1391-1396.

3 McIvor RA, Pizzichini E, Turner MO, Hussack P, Hargreave FE, Sears MR. Potential masking effects of salmeterol on airway inflammation in asthma. Am J Respir Crit Care Med 1998; 158: 924-930.

4 Mann M, Chowdhury B, Sullivan E, Nicklas R, Anthracite R, Meyer RJ. Serious asthma exacerbations in asthmatics treated with high-dose formoterol. Chest 2003; 124: 70-74.

5 Salpeter SR, Ormiston TM, Salpeter EE. Meta-analysis: respiratory tolerance to regular beta2-agonist use in patients with asthma. Ann Intern Med 2004; 140: 802-813.

6 Salpeter SR, Ormiston TM, Salpeter EE. Cardiovascular effects of beta agonists in patients with asthma and COPD: a meta-analysis. Chest 2004; 125: 2309-2321.
7 Cazzola M, Matera MG, Donner CF. Inhaled beta2adrenoceptor agonists: cardiovascular safety in patients with obstructive lung disease. Drugs 2005; 65: 1595-1610.

8 Kallergis EM, Manios EG, Kanoupakis EM, et al. Acute electrophysiologic effects of inhaled salbutamol in humans. Chest 2005; 127: 2057-2063.

9 Pearce N, Grainger J, Atkinson M, et al. Case-control study of prescribed fenoterol and death from asthma in New Zealand, 1977-81. Thorax 1990; 45: 170-175.

10 Grainger J, Woodman K, Pearce N, et al. Prescribed fenoterol and death from asthma in New Zealand, 19817: a further case-control study. Thorax 1991; 46: 105-111.

11 Spitzer WO, Suissa S, Ernst $\mathrm{P}$, et al. The use of beta-agonists and the risk of death and near death from asthma. N Engl J Med 1992; 326: 501-506.

12 Inman WH, Adelstein AM. Rise and fall of asthma mortality in England and Wales in relation to use of pressurised aerosols. Lancet 1969; 2: 279-285.

13 Nelson HS, Weiss ST, Bleecker ER, Yancey SW, Dorinsky PM, SMART Study Group. The Salmeterol Multicenter Asthma Research Trial: a comparison of usual pharmacotherapy for asthma or usual pharmacotherapy plus salmeterol. Chest 2006; 139: 15-26.

14 Castle W, Fuller R, Hall J, Palmer J. Serevent nationwide surveillance study: comparison of salmeterol with salbutamol in asthmatic patients who require regular bronchodilator treatment. BMJ 1993; 306: 1034-1037.

15 FitzGerald JM, Chapman KR, Della Cioppa G, et al. Sustained bronchoprotection, bronchodilatation, and symptom control during regular formoterol use in asthma of moderate or greater severity. The Canadian FO/OD1 Study Group. J Allergy Clin Immunol 1999; 103: 427-435.

16 Bensch G, Lapidus RJ, Levine BE, et al. A randomized, 12week, double-blind, placebo-controlled study comparing formoterol dry powder inhaler with albuterol metereddose inhaler. Ann Allergy Asthma Immunol 2001; 86: 19-27.

17 Wolfe J, Laforce C, Friedman B, et al. Formoterol, $24 \mu \mathrm{g}$ bid, and serious asthma exacerbations: similar rates compared with formoterol, $12 \mu \mathrm{g}$ bid, with and without extra doses taken on demand, and placebo. Chest 2006; 129: 27-38. 